\title{
Fermi surface instabilities in electronic Raman scattering of the metallic kagome lattice CsV3Sb5
}

\section{Dirk Wulferding ( $\nabla$ dirwulfe@snu.ac.kr)}

Seoul National University https://orcid.org/0000-0003-4279-2109

\section{Seungyeol Lee}

Chung-Ang University

\section{YoungSu Choi}

\section{Qiangwei Yin}

Renmin University of China

\section{Zhijun Tu}

Renmin University of China

\section{Chunsheng Gong}

Renmin University of China

Hechang Lei

Renmin University of China https://orcid.org/0000-0003-0850-8514

K.-Y. Choi

Sungkyunkwan University

\section{Article}

Keywords: fermi surface, charge density wave, electronic raman spectroscopy, QEP

Posted Date: September 3rd, 2021

DOI: https://doi.org/10.21203/rs.3.rs-846651/v1

License: (c) (i) This work is licensed under a Creative Commons Attribution 4.0 International License.

Read Full License 
1

\title{
Fermi surface instabilities in electronic Raman scattering of the metallic kagome lattice $\mathrm{CsV}_{3} \mathrm{Sb}_{5}$
}

\author{
Dirk Wulferding ${ }^{1,2,6 *}$, Seungyeol Lee ${ }^{3,6}$, Youngsu Choi ${ }^{4}$, Qiangwei Yin ${ }^{5}$, \\ Zhijun $\mathrm{Tu}^{5}$, Chunsheng Gong ${ }^{5}$, Hechang Lei ${ }^{5 *} \&$ Kwang-Yong Choi ${ }^{4 *}$ \\ ${ }^{1}$ Center for Correlated Electron Systems, \\ Institute for Basic Science, \\ Seoul 08826, Republic of Korea \\ ${ }^{2}$ Department of Physics and Astronomy, \\ Seoul National University, \\ Seoul 08826, Republic of Korea \\ ${ }^{3}$ Department of Physics, \\ Chung-Ang University, \\ Seoul 06974, Republic of Korea \\ ${ }^{4}$ Department of Physics, \\ Sungkyunkwan University, \\ Suwon 16419, Republic of Korea \\ ${ }^{5}$ Department of Physics and Beijing Key Laboratory of \\ Opto-electronic Functional Materials 86 Micro-nano Devices, \\ Renmin University of China, \\ Beijing 100872, China \\ ${ }^{6}$ These authors contributed equally: Dirk Wulferding, Seungyeol Lee \\ *email: dirwulfe@snu.ac.kr; hlei@ruc.edu.cn; choisky99@skku.edu
}


Understanding the link between a charge density wave (CDW) instability and superconductivity is a central theme of the $2 \mathrm{D}$ metallic kagome compounds $A \mathbf{V}_{3} \mathrm{Sb}_{5}(A=\mathbf{K}, \mathbf{R} \mathbf{b}$, and $\mathbf{C s})$. Using polarization-resolved electronic Raman spectroscopy, we shed light on Fermi surface fluctuations and electronic instabilities. We observe a quasielastic peak (QEP) whose spectral weight is progressively enhanced towards the superconducting transition. The QEP temperaturedependence reveals a steep increase in coherent in-plane charge correlations within the charge-density phase. In contrast, out-of-plane charge fluctuations remain strongly incoherent across the investigated temperature range. In-plane phonon anomalies appear at $T^{*} \sim 50 \mathrm{~K}$ in addition to right below $T_{\mathrm{CDW}} \sim 95 \mathrm{~K}$, while showing no apparent evidence of reduced symmetry at low temperatures. In conjunction with the consecutive phonon anomalies within the CDW state, our electronic Raman data unveil additional electronic instabilities that persist down to the superconducting phase, thereby offering a superconducting mechanism.

\section{INTRODUCTION}

Two-dimensional kagome metals are at the forefront of correlated topological physics as their electronic structure entails flat bands, van Hove singularities, and Dirac cones. The sought-after many-body states in kagome materials include topological superconductivity, unconventional charge density wave (CDW), fractional quantum Hall, and Majorana fermions $\underline{1}^{\underline{1} \underline{\underline{9}}}$.

The recently discovered kagome metals $A \mathrm{~V}_{3} \mathrm{Sb}_{5}(A=\mathrm{K}, \mathrm{Rb}$, and $\mathrm{Cs} ; P 6 / \mathrm{mmm}$ space group) have opened a new avenue toward establishing the relationship between CDW, anomalous Hall effect (AHE), and superconductivity (SC) $\underline{\underline{10}}$. In this V-based kagome family, an alkali metal layer and a $\mathrm{V}_{3} \mathrm{Sb}_{5}$ layer are alternately stacked, forming quasi-2D kagome layers of the $\mathrm{V}$ ions. $\mathrm{Cs}_{3} \mathrm{Sb}_{5}$ features $\mathbb{Z}_{2}$ band topology and a two-stage symmetry breaking: a CDW transition at $T_{\mathrm{CDW}} \sim 95 \mathrm{~K}$ with a subsequent superconducting transition at $T_{\mathrm{C}} \sim 2.7 \mathrm{~K}^{10} \underline{-14}$. The CDW order with inverse star-of-David pattern involves $2 \times 2$ and $1 \times 4$ charge orders 15,16 . The nesting of the Fermi surface assisted by the M-point van Hove singularity may provide a primary cause of the CDW transition $\frac{15}{2}$. Strikingly, a large AHE 
concurs with the CDW order despite the lack of long-range magnetic ordering $10,12,15,17-19$.

To account for spontaneous time-reversal symmetry breaking, orbital currents with different closed-loop patterns have been invoked $16,20-22$. Currently, there are opposing experimental reports on the existence of a chiral flux current phase and the field tunability of chiral charge order ${ }^{23}-25$. At low pressures, double-dome SC behavior is observed within the CDW phase and a new SC dome emerges at higher pressures where the CDW transition is completely repressed $26-30$. Aside from the rich pressure-induced SC phases, contradictory views exist on the SC pairing mechanism and symmetry. The residual linear term of thermal conductivity along with Majorana modes and pair-density wave observed by scanning tunneling microscopy (STM) favour unconventional nodal superconductivity $\underline{31} \underline{-33}$. Conversely, the magnetic penetration depth and the Hebel-Slichter coherence peak of ${ }^{51} \mathrm{~V} 1 / T_{1} T$ support an $s$-wave superconductor $\stackrel{34,35}{35}$ or multiband superconductivity with sign-preserving order parameter $\underline{36}-38$.

To pin down the nature of the SC phase, it is pivotal to elucidate Fermi surface instabilities below the CDW state and their connection to SC. In this work, we employ polarizationresolved Raman spectroscopy to probe low-energy electronic fluctuations and to evaluate their possible role in the superconducting pairing mechanism. We uncover an additional phonon anomaly and an electronic instability below $T_{\mathrm{CDW}}$, which may provide a gluing channel to superconductivity. The angular-dependent Raman data show a dominant $s$-wave character of the electronic correlations down to $T=5 \mathrm{~K}$, which constrains the possible pairing symmetry.

\section{RESULTS}

Electronic Raman scattering and phonons. To disentangle in-plane from out-ofplane dynamical electronic behavior, we performed temperature-dependent Raman measurements in two different scattering geometries, as sketched in Fig. 1a. In Fig. 1b, we show representative Raman spectra obtained at $T=5 \mathrm{~K}$ and $300 \mathrm{~K}$ in in-plane and out-of-plane polarizations. The Raman spectra comprise sharp, well-defined phonon peaks superimposed on a broad electronic background. We decompose the electronic Raman scattering into two contributions: a rather symmetric maximum centered around $17 \mathrm{meV}$ (shaded pale green) and a quasielastic peak (QEP) (shaded pale red). The former is assigned to an interband 

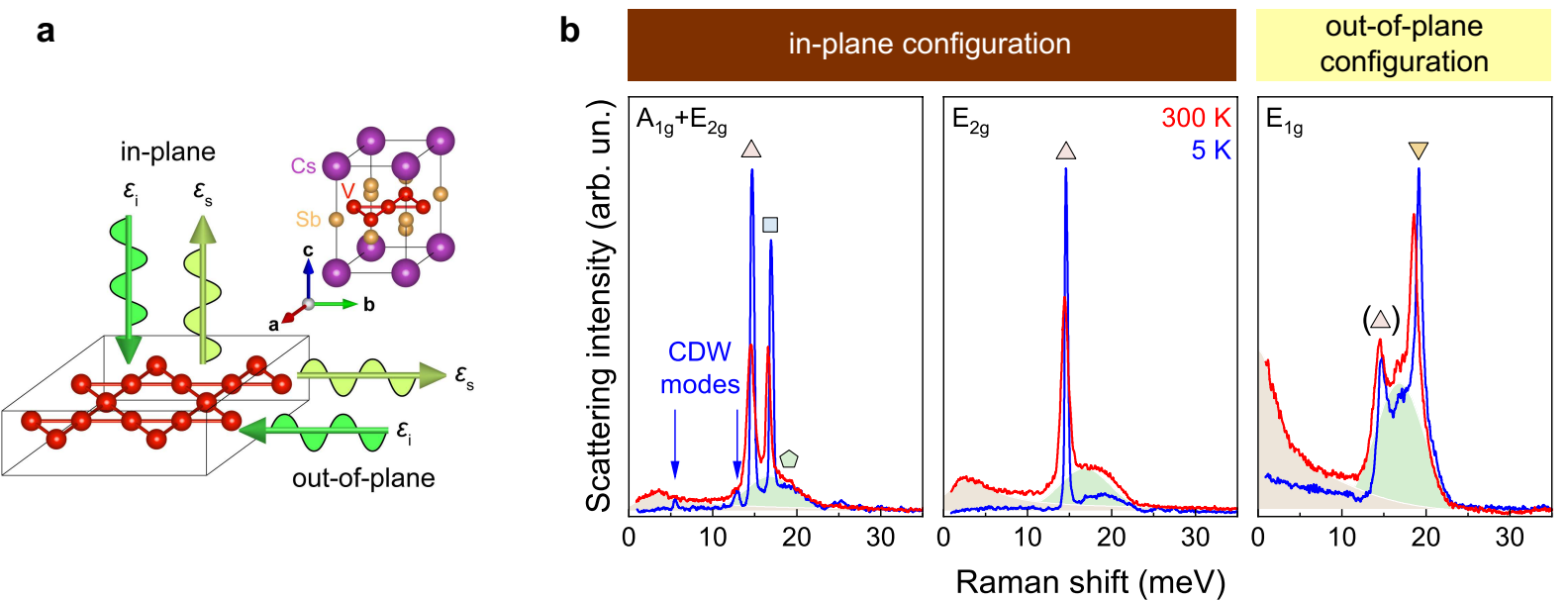

FIG. 1. Polarization-resolved Raman spectra of $\mathbf{C s V}_{3} \mathbf{S b}_{5}$. a Crystal structure of $\mathrm{CsV}_{3} \mathrm{Sb}_{5}$ and a sketch of the experimental Raman scattering geometries on a kagome lattice comprised of $\mathrm{V}$ atoms. The green arrows depict the incident and scattered light directions for in-plane and out-of-plane measurements. The shaded sine curves mark the direction of the electric field vector. b Raman spectra measured in three distinct symmetry channels $\mathrm{A}_{1 g}+\mathrm{E}_{2 g}, \mathrm{E}_{2 g}$, and $\mathrm{E}_{1 g}$ at two temperatures $T=300 \mathrm{~K}$ and $5 \mathrm{~K}$. The symbols mark symmetry-allowed optical phonons and a broad electronic background (green pentagon).

transition and the latter QEP pertains to a long-wavelength dynamical electronic response. The in-plane QEP forms a maximum at $2.5 \mathrm{meV}$, while the out-of-plane QEP is centered around zero energy. The difference of the QEP maximum between the in-plane and out-ofplane channels signifies anisotropic electronic scattering processes.

Besides, we observe two Raman-active $\mathrm{A}_{1 g}$ and $\mathrm{E}_{2 g}$ phonons at $17.9 \mathrm{meV}$ and $14.7 \mathrm{meV}$, respectively, for in-plane scattering geometries. In the out-of-plane configuration, a single $\mathrm{E}_{1 g}$ mode appears at $19.2 \mathrm{meV}$, in accordance with the Raman tensors (see Methods). We further note that a weak remnant of the $\mathrm{A}_{1 g}$ phonon can be seen in the $\mathrm{E}_{1 g}$ channel (the triangle in brackets). Since a fresh cut along the $c$ axis was performed at a random in-plane orientation, a finite leakage from the $\mathrm{A}_{1 g}$ channel might be inevitable. This is contrasted by no leakage of the $\mathrm{E}_{1 g}$ phonon in our in-plane measurements.

Thermal evolution of the Raman response and conductivity. In Fig. 2a, we plot the temperature dependence of the Bose-corrected Raman response $\chi^{\prime \prime}(\omega, T)$ (see Supplementary Fig. S1 for the as-measured Raman intensity). With decreasing temperature, the in-plane low-energy $\chi_{\mathrm{ip}}^{\prime \prime}(\omega, T)$ displays a gradual buildup of its spectral weight below 22 
a

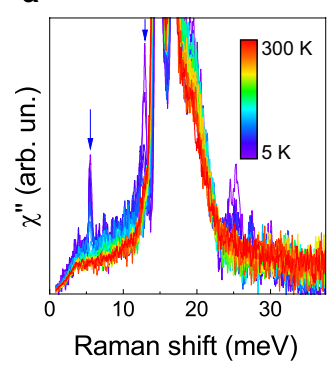

e

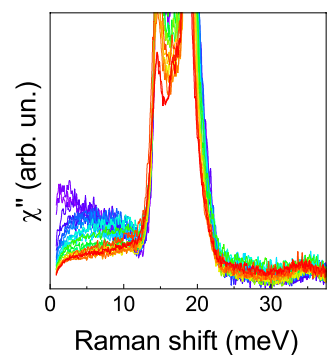

b

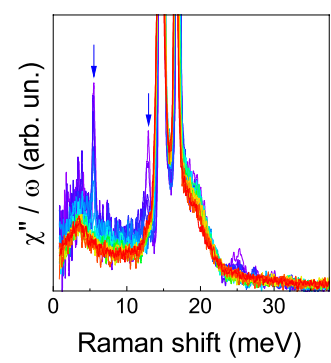

f

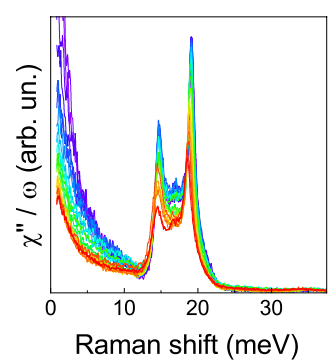

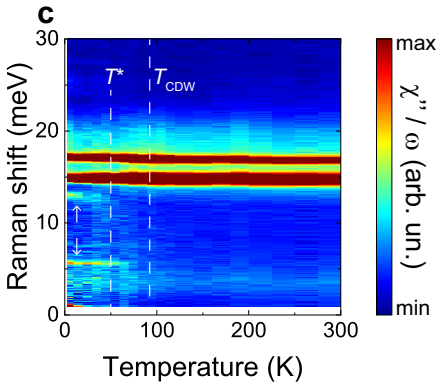

d
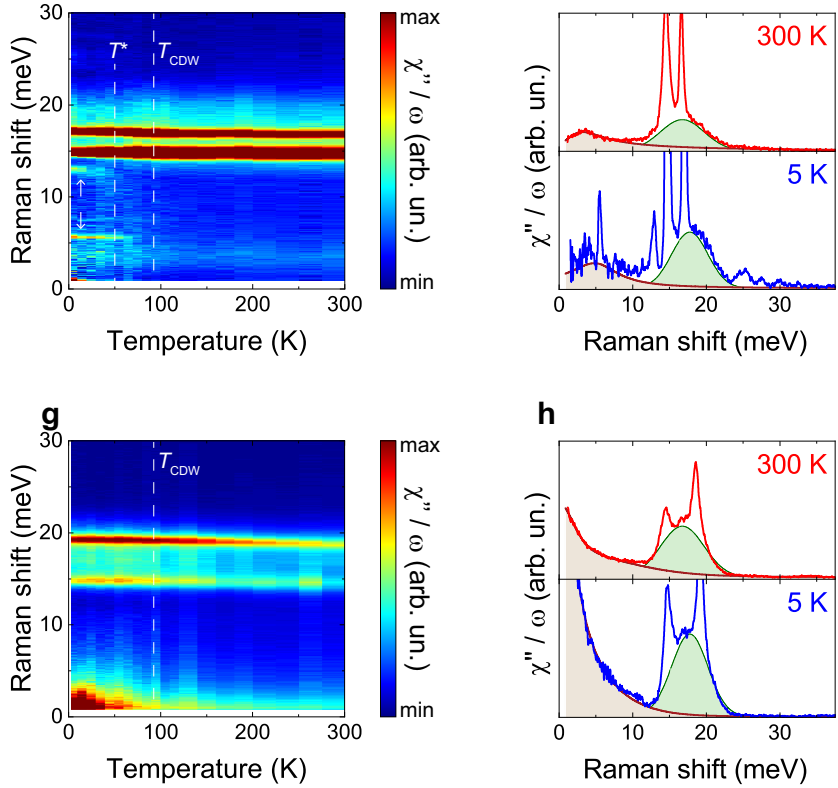

$\mathbf{h}$

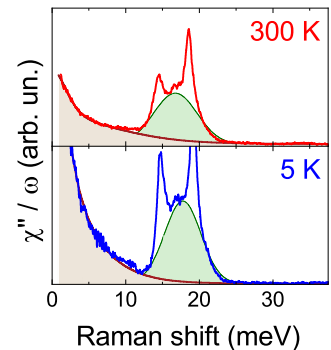

FIG. 2. Electronic Raman scattering in $\mathbf{C s V}_{3} \mathbf{S b}_{5}$. a Bose-corrected Raman spectra $\chi^{\prime \prime}(\omega)$ and b Raman conductivity $\chi^{\prime \prime}(\omega) / \omega$ measured between $5 \mathrm{~K}$ and $300 \mathrm{~K}$ in an in-plane scattering channel. c Color plot of in-plane $\chi^{\prime \prime}(\omega) / \omega$ vs temperature. The white arrows point towards CDW-induced phonons. d Modeling of the in-plan Raman conductivity in terms of the low-energy quasielastic peak (pale red) and an electronic continuum at finite energies (pale green) e-h Out-of-plane $\chi^{\prime \prime}(\omega)$, $\chi^{\prime \prime}(\omega) / \omega$, and a color contour map of $\chi^{\prime \prime}(\omega) / \omega$ as a function of temperature, together with fits of the electronic Raman response, respectively.

meV. The Raman conductivity $\chi_{\mathrm{ip}}^{\prime \prime}(\omega, T) / \omega$, shown in Fig. 2b, highlights the thermal growth of low-energy electronic scattering. Evident from the color contour plot of $\chi_{\mathrm{ip}}^{\prime \prime}(\omega, T) / \omega$ in Fig. 2c, the QEP is slightly enhanced for temperatures below $\approx T_{\mathrm{CDW}}$. Besides, CDWinduced phonons appear at $5.5 \mathrm{meV}$ and $12 \mathrm{meV}$ (see Fig. 4d and below). We analyze the Raman conductivity by a sum of two individual electronic excitations as demonstrated for $T=300 \mathrm{~K}$ and $5 \mathrm{~K}$ in Fig. 2d. The low-energy QEP is described by a collision-dominated model applied to the Bose corrected Raman response $\chi_{\mathrm{QEP}}^{\prime \prime}(\omega) \sim A \Gamma /\left(\omega^{2}+\Gamma^{2}\right)$, where $A$ is the scattering amplitude and $\Gamma(T)$ is the scattering rate. We model the higher-energy excitation by a Gaussian profile.

In contrast to the in-plane behavior, the out-of-plane $\chi_{\mathrm{op}}^{\prime \prime}(\omega, T)$, shown in Fig. 2e, gains progressively in low-energy spectral weight and shifts to zero frequency with decreasing temperature. $\chi_{\mathrm{op}}^{\prime \prime}(\omega, T) / \omega$ and its color plot in Fig. 2f,g visualize the steep growth of QEP 

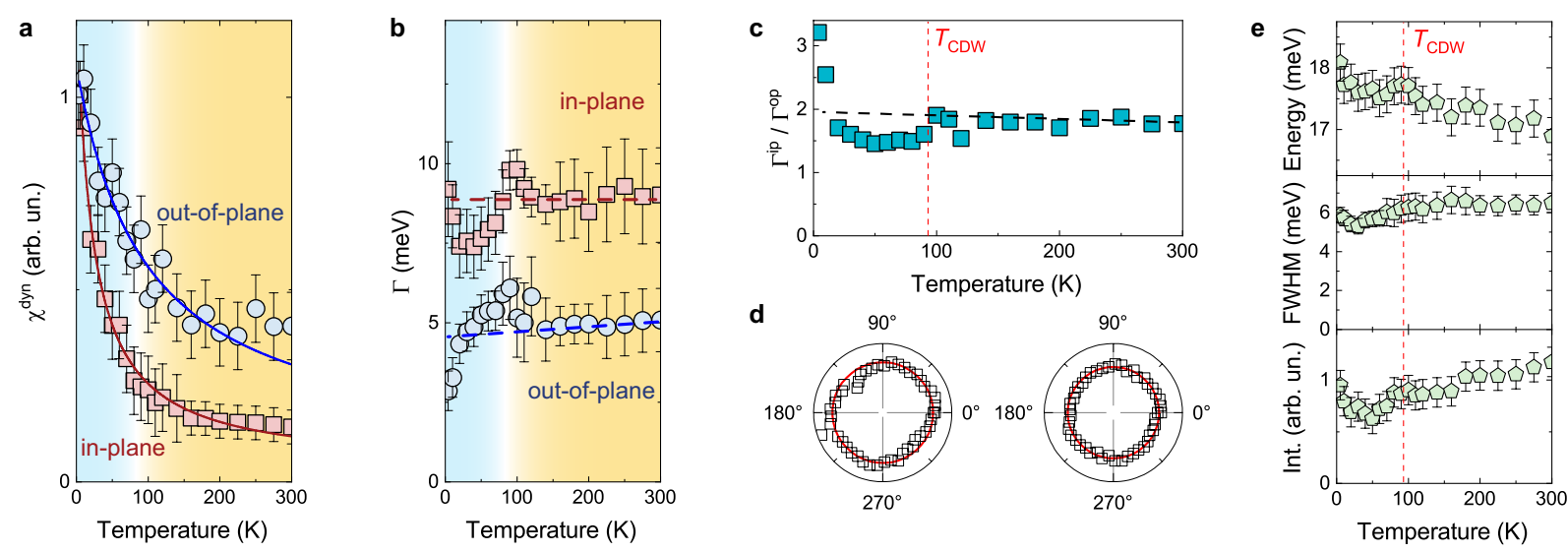

FIG. 3. Raman susceptibility, scattering rate and the symmetry of electronic scattering. a Raman susceptibility derived from in-plane and out-of-plane $\chi^{\prime \prime}(\omega) / \omega$. The blue and red lines are fits to a Curie-Weiss behavior. b Scattering rate extracted from fittings of the low-energy quasielastic peak to a collision-dominated model (see text) measured in in-plane (red squares) and out-of-plane (blue circles) channels. The dashed lines denote a linear temperature dependence. c The ratio of in-plane vs. out-of-plane scattering rate as a function of temperature. The dashed black line marks the close-to-linear temperature-dependence above $T_{\mathrm{CDW}}$. d Angular dependence of the in-plane electronic Raman scattering intensity measured at $T=5 \mathrm{~K}$ (left) and $T=80$ K (right). e Temperature-dependent energy, linewidth, and intensity of the electronic continuum centered around $17 \mathrm{meV}$.

as the temperature is lowered through $T_{\mathrm{CDW}}$. Overall, the divergent or steady increase of $\chi^{\prime \prime}(\omega, T)$ below $T_{\mathrm{CDW}}$, depending on the scattering channels, alludes to a secondary electronic instability of the pre-existing CDW ordered state and anisotropic scattering mechanism. Fig. $2 \mathrm{~h}$ shows the decomposition of the out-of-plane electronic Raman conductivity into QEP and broad continuum.

Raman susceptibility and symmetry of electronic scattering. To shed light on the divergent QEP behavior and its link to an electronic instability, we analyze the dynamic Raman susceptibility $\chi^{\mathrm{dyn}}$ at zero frequency using the Kramers-Kronig relation $\underline{39}$ :

$$
\chi^{\mathrm{dyn}}=\lim _{\omega \rightarrow 0} \chi(k=0, \omega) \sim \int_{0}^{\infty} \frac{\chi^{\prime \prime}(\omega)}{\omega} \mathrm{d} \omega .
$$

$\chi^{\mathrm{dyn}}(T)$ allows us to access dynamic electronic fluctuations for a given symmetry channel, thereby we may quantify in-plane vs. out-of-plane electronic correlations. $\chi^{\text {dyn }}(T)$ is ob- 
tained by integrating $\chi^{\prime \prime}(\omega) / \omega$ from $1 \mathrm{meV}$ up to $50 \mathrm{meV}$ and plotted in Fig. 3a for both scattering channels. Over the entire measured temperature range of $T=5-300 \mathrm{~K}$, the temperature evolution of $\chi^{\mathrm{dyn}}(T)$ is described by a Curie-Weiss law $\chi^{\mathrm{dyn}}(T) \propto \frac{1}{T-T_{\mathrm{CW}}}$ with the Curie-Weiss temperature $T_{\mathrm{CW}}$. The lacking divergence of $\chi^{\mathrm{dyn}}(T)$ at $T_{\mathrm{CDW}}$ alludes to an unconventional CDW order. Rather, the fact that the Curie-like increase of $\chi^{\text {dyn }}(T)$ persists to the SC state supports the notion of an incipient electronic instability in addition to the CDW transition. From the in-plane $\chi^{\mathrm{dyn}}(T)$, we extract $T_{\mathrm{CW}}^{\mathrm{ip}} \approx-22 \mathrm{~K}$, while the out-of-plane $\chi^{\mathrm{dyn}}(T)$ yields $T_{\mathrm{CW}}^{\mathrm{op}} \approx-96 \mathrm{~K}$, i.e., very close to $T_{\mathrm{CDW}}$ (see Supplementary Fig. S2 for $1 / \chi^{\text {dyn }}$ plots).

Several comments on the distinct $T_{\mathrm{CW}}$ values are in order. Given that $T_{\mathrm{CW}}^{\mathrm{ip}}, T_{\mathrm{CW}}^{\mathrm{op}} \leq T_{\mathrm{CDW}}$, an instability of the Fermi surface may be inherited from the CDW reconstructed Fermi surface or a second CDW instability occurs in the primary CDW ordered state. On the other hand, $T_{\mathrm{CW}}^{\mathrm{ip}} \ll T_{\mathrm{CW}}^{\mathrm{op}} \approx T_{\mathrm{CDW}}$ suggests that another in-plane electronic instability emerges well below $T_{\mathrm{CDW}}$ (see below) and may be inextricably tied to the SC formation.

Next, we discuss the QEP fitting parameters as a function of temperature. Detailed information on the individual fits is given in the Supplementary (Figs. S3 and S4). Since $A(T)$ and $\chi^{\text {dyn }}(T)$ convey essentially the same information, we focus on $\Gamma(T)$, plotted in Fig. 3b. Both the in-plane and out-of-plane $\Gamma(T)$ exhibit a linear decrease at high temperatures. On approaching $T_{\mathrm{CDW}}$, both quantities slightly increase with a clear peak at about $T_{\mathrm{CDW}}$. The critical-like slowing down of CDW related excitations supports that the CDW order is mainly driven by electronic correlations. For lower temperatures, the out-of-plane $\Gamma^{\mathrm{op}}(T)$ tends to drop to zero as $T \longrightarrow 0 \mathrm{~K}$. In stark contrast, the in-plane $\Gamma^{\mathrm{ip}}(T)$ shows a strong upturn upon approaching $T_{c}$. This disparate behavior is highlighted in Fig. 3c, where the ratio of $\Gamma^{\mathrm{ip}} / \Gamma^{\mathrm{op}}$ evidences the increasingly anisotropic electronic correlations prior to the SC phase. In a weak coupling limit, the QEP can be understood as the Drude contribution to $\chi^{\prime \prime}(\omega)$ and $\Gamma(T)$ undergoes a renormalization by $\Gamma(T) \propto \Gamma_{0}\left(T-T_{0}\right)$ with the single-particle scattering rate $\Gamma_{0} \underline{39}$. We find $\Gamma_{0}^{\mathrm{ip}} / \Gamma_{0}^{\mathrm{op}} \approx 2$ above $T_{\mathrm{CDW}}$. That is, the electronic fluctuations in the in-plane are twice as slow as in the out-of-plane channel prior to the CDW transition. The remarkable observation is the steep increase of $\Gamma_{0}^{\text {in }} / \Gamma_{0}^{\text {op }}$ as $T \longrightarrow T_{c}$, which goes hand in hand with the divergent $\chi^{\text {dyn }}(T)$.

To address the issue of in-plane anisotropies and possible symmetry lowering, we showcase angular plots of the in-plane electronic Raman scattering intensity measured at $T=5 \mathrm{~K}$ 

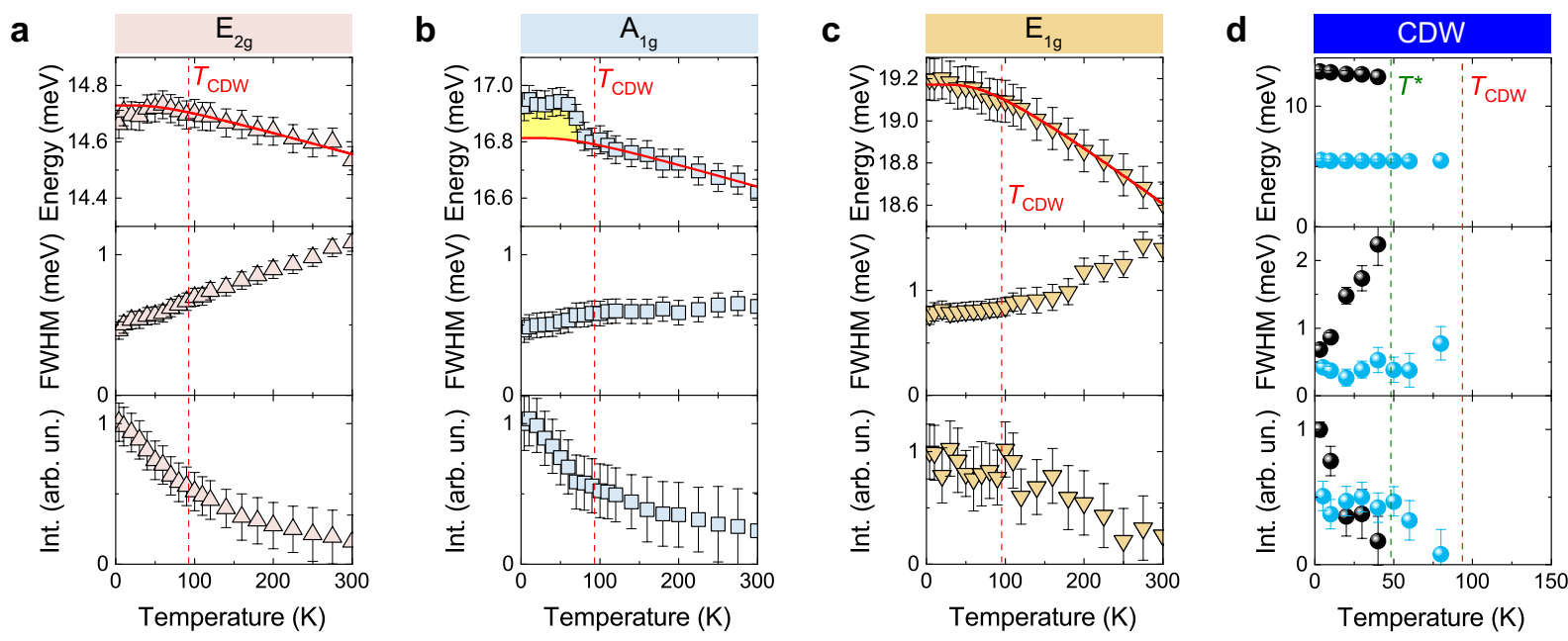

FIG. 4. Phonon dynamics in $\mathbf{C s V}_{3} \mathbf{S b}_{5}$. Temperature-dependent phonon energy, linewidth, and normalized intensity of $\mathbf{a}$ the in-plane $\mathrm{E}_{2 g}$ phonon, $\mathbf{b}$ the in-plane $\mathrm{A}_{1 g}$ phonon, $\mathbf{c}$ the out-of-plane $\mathrm{E}_{1 g}$ phonon, and $\mathbf{d}$ two CDW-induced modes. The solid red lines are fits to an anharmonic decay model (see Methods for details). The vertical dashed lines mark the CDW transition temperature and a second CDW mode onset temperature $T^{*}$.

(left panel Fig. 3d) and at $T=80 \mathrm{~K}$ (right panel Fig. 3d). Within our signal-noise ratio, we find no apparent angular dependence at both temperatures. This observation is at odds with a symmetry reduction of the electronic response through the CDW and approaching the SC state. Assuming that the secondary electronic instabilities below $T_{\mathrm{CDw}}$ give rise to $\mathrm{SC}$, our angular variation data impose $s$-wave symmetry to the SC pairing. Nonetheless, we cannot exclude the possibility that a limited spectral resolution can obscure weakly broken rotational symmetry. Shown in Fig. 3e is the thermal evolution of the mid-energy electronic scattering signal centered around $17 \mathrm{meV}$. With decreasing temperature, all three quantities (energy, linewidth, and intensity) evidence a subtle discontinuous behavior around $T_{\mathrm{CDW}}$. This may reflect the slight band modifications accompanying CDW.

CDW induced phonon anomaly. To elucidate phonon anomalies associated with the CDW transition, we present the phonon parameters (energy, linewidth, and intensity) as a function of temperature for all three phonons in Figs. 4a-c. The CDW transition involves no discernible effect on the out-of-plane $\mathrm{E}_{1 g}$ phonon, while a minute softening can be seen in the energy of the in-plane $\mathrm{E}_{2 g}$ phonon. In contrast, the in-plane $\mathrm{A}_{1 g}$ phonon follows conventional anharmonic behavior only down to $T_{\mathrm{CDW}}$. At lower temperatures, it pronouncedly hardens, 
together with a discontinuous increase in intensity. In addition, two CDW modes of $\mathrm{A}_{1 g}$ symmetry are activated by the charge-order-induced lattice modulations (as marked by the blue arrows in Fig. 1b and detailed in Fig. 4d). We recall that the CDW-induced modes are observed only in the in-plane polarization (see the vertical arrows in Fig. 1), indicative of the dominant two-dimensional nature of charge orders. Remarkably, the high-energy mode located around $12 \mathrm{meV}$, and thereby close to the $\mathrm{A}_{1 g}$ phonon develops on the left shoulder of the electronic continuum below $T^{*} \sim 50 \mathrm{~K}$. It dramatically loses in intensity and broadens significantly well below $T_{\mathrm{CDW}}$. Conversely, the lower energy CDW mode vanishes just below $T_{\mathrm{CDW}}$, with its intensity following an order-parameter-like behavior. The rapid repression and damping of the $12 \mathrm{meV}$ mode indicates its strong susceptibility to electronic fluctuations. The two characteristic temperatures $T^{*}$ and $T_{\mathrm{CDW}}$ imply the presence of an extra electronic instability or a charge modulation deep inside the CDW state. Indeed, STM and ${ }^{51} \mathrm{~V}$ NMR studies of $\mathrm{CsV}_{3} \mathrm{Sb}_{5}$ reveal another charge modulation at $40 \mathrm{~K}$, which is interpreted in terms of a $4 a_{0}$ charge modulation pattern or a roton pair density wave $\underline{33,40}$.

\section{DISCUSSION}

Using polarization-resolved electronic Raman scattering, we have detected a QEP that stems from long-wavelength electronic density fluctuations. The salient observations are that the QEP is continuously enhanced in $\chi^{\prime \prime}$ upon approaching the SC state and that it features an anisotropic scattering rate between the intra- and inter-plane channels. The strong enhancement of the QEP for $T<T_{\mathrm{CDW}}$ implies the presence of dynamical charge fluctuations in the background of the long-range charge orders in the CDW state and, thereby, raises the possibility of their link to SC.

The QEP associated susceptibilities show a Curie-like divergence as $T \longrightarrow T_{c}$. The divergent behavior of $\chi^{\mathrm{dyn}}(T)$ is reminiscent of a Pomeranchuk instability that is responsible for a nematic state of the Fe-based superconductors $\stackrel{41}{\underline{4}}$. The presence of Fermi surface instabilities is further supported by the steeply increasing $\Gamma_{0}^{\mathrm{ip}} / \Gamma_{0}^{\mathrm{op}}$ at low temperatures. Recent theoretical studies have put forward Pomeranchuk fluctuations of a reconstructed Fermi surface or smectic bond-density-wave fluctuations as SC pairing mechanisms $\underline{42,43}$. Our angulardependent Raman scattering study of the QEP closely resembles an isotropic $s$-wave character within the CDW phase (see Fig. 3d, and Supplementary Fig. S5), which seems to be 
at odds with the original proposal that favors a $d$-wave Pomeranchuk channel. Nonetheless, both our phonon spectra and the ${ }^{51} \mathrm{~V}$ NMR line independently demonstrate the occurrence of another charge modulation on top of the inverse star-of-David pattern ${ }^{40}$. This hints towards a weak (possibly twofold) anisotropy, which could be a vestige of an emerging symmetry breaking within the superconducting phase. Putting aside the exact origin of the electronic instabilities, we expect dominant $s$-wave $\mathrm{SC}$, if the observed electronic instabilities at $T>T_{c}$ are responsible for the gluing mechanism of Cooper pairs. Future Raman studies within the superconducting phase and under hydrostatic pressure are envisaged to identify the origin of the QEP unambiguously and its relation to SC as the CDW and SC display a distinct pressure evolution.

In summary, our Raman scattering study of $\mathrm{CsV}_{3} \mathrm{Sb}_{5}$ discloses secondary electronic instabilities in the CDW state, which arise from either Fermi surface fluctuations or another type of weak density wave or charge order. Their $s$-wave symmetry with tantalizing rotational symmetry breaking and two temperature scales will pose constraints on viable theories of establishing the relationship between CDW and SC.

\section{METHODS}

Sample preparation. Single crystals of $\mathrm{CsV}_{3} \mathrm{Sb}_{5}$ were grown from Cs ingot (purity 99.9 \%), V powder (purity $99.9 \%$ ) and Sb grains (purity 99.999\%) using the self-flux method, similar to the growth of $\mathrm{RbV}_{3} \mathrm{Sb}_{5} \stackrel{14}{ }$. The eutectic mixture of $\mathrm{CsSb}_{\mathrm{S}}$ and $\mathrm{CsSb}_{2}$ is mixed with $\mathrm{VSb}_{2}$ to form a composition with approximately $50 \% \mathrm{Cs}_{x} \mathrm{Sb}_{y}$ and $50 \% \mathrm{VSb}_{2}$. The mixture was placed into an alumina crucible and sealed in a quartz ampoule under a partial argon atmosphere. The sealed quartz ampoule was heated to $1273 \mathrm{~K}$ for $12 \mathrm{~h}$ and soaked there for $24 \mathrm{~h}$. It was subsequently cooled down to $1173 \mathrm{~K}$ at $50 \mathrm{~K} / \mathrm{h}$ and further to $923 \mathrm{~K}$ at a slower rate. Finally, the ampoule was removed from the furnace and decanted with a centrifuge to separate $\mathrm{CsV}_{3} \mathrm{Sb}_{5}$ single crystals from the flux. The obtained crystals have a typical size of $2 \times 2 \times 0.02 \mathrm{~mm}^{3}$.

Raman spectroscopy. To avoid degradation of the sample surface, a freshly cleaved single crystal of $2 \mathrm{~mm} \times 2 \mathrm{~mm} \times 0.2 \mathrm{~mm}$ was mounted onto the cold-finger of a heliumflow cryostat inside an Ar atmosphere glove box. Raman spectroscopic measurements were carried out using a $\lambda=561 \mathrm{~nm}$ laser (Oxxius LCX) and a Princeton Instruments TriVista 
spectrometer equipped with a volume Bragg grating notch filter (Optigrate; low-energy cutoff: $6 \mathrm{~cm}^{-1}$ ) and a liquid-Nitrogen cooled CCD (PyLoN eXcellon). The incident laser with a spot diameter of about $5 \mu \mathrm{m}$ was kept at a power level of $0.3 \mathrm{~mW}$ to minimize heating effects.

Analysis of phonons. $\mathrm{CsV}_{3} \mathrm{Sb}_{5}$ belongs to the hexagonal space group $P 6 / \mathrm{mmm}$, where the ions are located at the following Wyckoff positions: $\mathrm{Cs}-1 a, \mathrm{~V}-3 g, \mathrm{Sb} 1-1 b, \mathrm{Sb} 2-4 h \underline{10}$. This leads to a total of three Raman-active modes $\mathrm{A}_{1 g}+\mathrm{E}_{1 g}+\mathrm{E}_{2 g}$, with their corresponding Raman tensors

$\mathrm{A}_{1 g}=\left(\begin{array}{lll}a & 0 & 0 \\ 0 & a & 0 \\ 0 & 0 & b\end{array}\right), \mathrm{E}_{1 g}=\left(\begin{array}{lll}0 & 0 & 0 \\ 0 & 0 & c \\ 0 & c & 0\end{array}\right), \mathrm{E}_{2 g}=\left(\begin{array}{ccc}d & 0 & 0 \\ 0 & -d & 0 \\ 0 & 0 & 0\end{array}\right), \mathrm{E}_{2 g}=\left(\begin{array}{ccc}0 & -d & 0 \\ -d & 0 & 0 \\ 0 & 0 & 0\end{array}\right)$

The temperature dependence of the phonon energies has been approximated by a cubic anharmonic, second-order model $\underline{44}^{4}$ :

$$
\omega(T)=\omega_{0}-A \cdot\left(1+\frac{2}{\exp \left(\hbar \omega_{0} / 2 k_{B} T\right)-1}\right)
$$

where $\omega_{0}$ is the bare phonon energy at $T=0$, and $A$ is a fitting constant.

In metals electronic Raman scattering probes the long wavelength dynamical charge response in the symmetry channel $i$ : $S_{i}(\omega)=\left\langle\rho_{i}^{\dagger}(\omega) \rho_{i}(\omega)\right\rangle$, where $\omega$ is the Raman shift and $\rho_{i}$ is the electronic charge density. To derive the associated susceptibility, we first obtain the imaginary Raman response in the dynamical limit $\chi^{\prime \prime}(\omega)$ from as-measured Raman intensity $I(\omega)$ via the fluctuation-dissipation theorem by $I(\omega)=[1+n(\omega)] \chi^{\prime \prime}(\omega)$. Here, $[1+n(\omega)]=1 /\left(1-\mathrm{e}^{-\hbar \omega / k_{B} T}\right)$ is the Bose factor.

\section{ACKNOWLEDGMENTS}

We thank Inkyung Song and Tae-Yeong Park for important experimental support. This work was supported by the National Research Foundation (NRF) of Korea (Grants No. 2020R1A2C3012367, and No. 2020R1A5A1016518) and by the Institute for Basic Science (Grant No. IBS-R009-Y3). The work at SKKU was supported by the National Research Foundation (NRF) of Korea (Grants No. 2020R1A2C3012367, and No. 2020R1A5A1016518). H.C.L. was supported by National Natural Science Foundation of 
China (Grant No. 11822412 and 11774423), Ministry of Science and Technology of China (Grant No. 2018YFE0202600 and 2016YFA0300504) and Beijing Natural Science Foundation (Grant No. Z200005).

\section{AUTHOR CONTRIBUTIONS}

K.-Y.C. conceived and designed the experiments. Q.Y., Z.T., C.G., and H.L. synthesized the single crystal. D.W., S.L., and Y.C. performed Raman spectroscopic experiments. K.-Y.C., D.W., S.L., and Y.C. analyzed the data. D.W., K.-Y.C, and S.L. wrote the manuscript with input from all authors. All authors discussed the results and commented on the manuscript.

Competing interests: The authors declare no competing interests. Data and materials availability: All data needed to evaluate the conclusions in the paper are present in the paper and/or the Supplementary Materials. All data are available from the corresponding authors upon reasonable request.

1 Ko, W.-H., Lee, P. A. \& Wen, X.-G. Doped kagome system as exotic superconductor. Phys. Rev. B 79, 214502 (2009).

2 Guo, H.-M. \& Franz, M. Topological insulator on the kagome lattice. Phys. Rev. B 80, 113102 (2009).

3 Rüegg, A. \& Fiete, G. A. Fractionally charged topological point defects on the kagome lattice. Phys. Rev. B 83, 165118 (2011).

4 Wang, W.-S., Li, Z.-Z., Xiang, Y.-Y. \& Wang, Q.-H. Competing electronic orders on kagome lattices at van Hove filling. Phys. Rev. B 87, 115135 (2013).

5 Kiesel, M. L., Platt, C. \& Thomale, R. Unconventional Fermi Surface Instabilities in the Kagome Hubbard Model. Phys. Rev. Lett. 110, 126405 (2013).

6 Mazin, I. I. et al. Theoretical prediction of a strongly correlated Dirac metal. Nat. Commun. 5, $4261(2014)$.

7 Nakatsuji, S., Kiyohara, N. \& Higo, T. Large anomalous Hall effect in a non-collinear antiferromagnet at room temperature. Nature 527, 212 (2015). 
8 Ye, L. et al. Massive Dirac fermions in a ferromagnetic kagome metal. Nature 555, 638 (2018).

9 Yin, J.-X. et al. Quantum-limit Chern topological magnetism in $\operatorname{TbMn}_{6} \mathrm{Sn}_{6}$. Nature 583, 533 (2020).

10 Ortiz, B. R. et al. New kagome prototype materials: discovery of $\mathrm{KV}_{3} \mathrm{Sb}_{5}, \mathrm{RbV}_{3} \mathrm{Sb}_{5}$, and $\mathrm{CsV}_{3} \mathrm{Sb}_{5}$. Phys. Rev. Mater. 3, 094407 (2019).

11 Ortiz, B. R. et al. $\mathrm{CsV}_{3} \mathrm{Sb}_{5}$ : A Z $\mathrm{Z}_{2}$ Topological Kagome Metal with a Superconducting Ground State. Phys. Rev. Lett. 125, 247002 (2020).

12 Yang, S.-Y. et al. Giant, unconventional anomalous Hall effect in the metallic frustrated magnet candidate, $\mathrm{KV}_{3} \mathrm{Sb}_{5}$. Sci. Adv. 6, eabb6003 (2020).

13 Ortiz, B. R. et al. Superconductivity in the $\mathrm{Z}_{2}$ kagome metal $\mathrm{KV}_{3} \mathrm{Sb}_{5}$. Phys. Rev. Mater. 5, 034801 (2021).

14 Yin, Q. et al. Superconductivity and Normal-State Properties of Kagome Metal RbV $\mathrm{Sb}_{5}$ Single Crystals. Chin. Phys. Lett. 38, 037403 (2021).

15 Jiang, Y.-X. et al. Unconventional chiral charge order in kagome superconductor $\mathrm{KV}_{3} \mathrm{Sb}_{5}$. Nat. Mater. https://doi.org/10.1038/s41563-021-01034-y (2021).

16 Tan, H., Liu, Y., Wang, Z. \& Yan, B. Charge Density Waves and Electronic Properties of Superconducting Kagome Metals. Phys. Rev. Lett. 127, 046401 (2021).

$17 \mathrm{Yu}, \mathrm{F}$. H. et al. Concurrence of anomalous Hall effect and charge density wave in a superconducting topological kagome metal. Phys. Rev. B 104, L041103 (2021).

$18 \mathrm{Li}, \mathrm{H}$. et al. Rotation symmetry breaking in the normal state of a kagome superconductor $\mathrm{KV}_{3} \mathrm{Sb}_{5}$. Preprint at https://arxiv.org/abs/2104.08209 (2021).

19 Kenney, E. M., Ortiz, B. R, Wang, C., Wilson, S. D. \& Graf, M. J. Absence of local moments in the kagome metal $\mathrm{KV}_{3} \mathrm{Sb}_{5}$ as determined by muon spin spectroscopy. J. Phys.: Condens. Matter 33, 235801 (2021).

20 Feng, X., Jiang, K., Wang, Z. \& Hu, J. Chiral flux phase in the Kagome superconductor $\mathrm{AV}_{3} \mathrm{Sb}_{5}$. Sci. Bull. 66, 1384 (2021).

21 Denner, M. M., Thomale, R. \& Neupert, T. Analysis of charge order in the kagome metal $A \mathrm{~V}_{3} \mathrm{Sb}_{5}(A=\mathrm{K}, \mathrm{Rb}, \mathrm{Cs})$. Preprint at https://arxiv.org/abs/2103.14045 (2021).

22 Park, T., Ye, M. \& Balents, L. Electronic instabilities of kagome metals: Saddle points and Landau theory. Phys. Rev. B 104, 035142 (2021).

23 Mielke III, C. et al. Time-reversal symmetry-breaking charge order in a correlated kagome 
superconductor. Preprint at https://arxiv.org/abs/2106.13443 (2021).

$24 \mathrm{Yu}, \mathrm{L}$. et al. Evidence of a hidden flux phase in the topological kagome metal $\mathrm{CsV}_{3} \mathrm{Sb}_{5}$. Preprint at https://arxiv.org/abs/2107.10714 (2021).

$25 \mathrm{Li}, \mathrm{H}$. et al. No indication of chiral flux current in the topological kagome metal $\mathrm{CsV}_{3} \mathrm{Sb}_{5}$. Preprint at https://arxiv.org/abs/2107.11326 (2021).

$26 \mathrm{Yu}, \mathrm{F}$. H. et al. Unusual competition of superconductivity and charge-density-wave state in a compressed topological kagome metal. Nat. Commun. 12, 3645 (2021).

27 Chen, K. Y. et al. Double Superconducting Dome and Triple Enhancement of $T_{c}$ in the Kagome Superconductor $\mathrm{CsV}_{3} \mathrm{Sb}_{5}$ under High Pressure. Phys. Rev. Lett. 126, 247001 (2021).

$28 \mathrm{Du}, \mathrm{F}$. et al. Pressure-induced double superconducting domes and charge instability in the kagome metal $\mathrm{KV}_{3} \mathrm{Sb}_{5}$. Phys. Rev. B 103, L220504 (2021).

29 Zhang, Z. et al. Pressure-induced reemergence of superconductivity in the topological kagome metal $\mathrm{CsV}_{3} \mathrm{Sb}_{5}$. Phys. Rev. B 103, 224513 (2021).

30 Chen, X. et al. Highly Robust Reentrant Superconductivity in $\mathrm{CsV}_{3} \mathrm{Sb}_{5}$ under Pressure. Chinese Phys. Lett. 38, 057402 (2021).

31 Zhao, C. C. et al. Nodal superconductivity and superconducting domes in the topological Kagome metal $\mathrm{CsV}_{3} \mathrm{Sb}_{5}$. Preprint at http://arxiv.org/abs/2102.08356 (2021).

32 Liang, Z. et al. Three-Dimensional Charge Density Wave and Surface-Dependent Vortex-Core States in a Kagome Superconductor $\mathrm{CsV}_{3} \mathrm{Sb}_{5}$. Phys. Rev. X 11, 031026 (2021).

33 Chen, H. et al. Roton pair density wave and unconventional strong-coupling superconductivity in a topological kagome metal. Preprint at https://arxiv.org/abs/2103.09188 (2021).

34 Duan, W. et al. Nodeless superconductivity in the kagome metal $\mathrm{CsV}_{3} \mathrm{Sb}_{5}$. Sci. China-Phys. Mech. Astron. 64, 107462 (2021).

$35 \mathrm{Mu}, \mathrm{C}$. et al. S-Wave Superconductivity in Kagome Metal $\mathrm{CsV}_{3} \mathrm{Sb}_{5}$ Revealed by ${ }^{121 / 123} \mathrm{Sb}$ NQR and ${ }^{51}$ V NMR Measurements. Chinese Phys. Lett. 38, 077402 (2021).

$36 \mathrm{Xu}, \mathrm{H} .-\mathrm{S}$. et al. Multiband superconductivity with sign-preserving order parameter in kagome superconductor $\mathrm{CsV}_{3} \mathrm{Sb}_{5}$. Preprint at https://arxiv.org/abs/2104.08810 (2021).

37 Nakayama, K. et al. Multiple Energy Scales and Anisotropic Energy Gap in the Charge-Density-Wave Phase of Kagome Superconductor $\mathrm{CsV}_{3} \mathrm{Sb}_{5}$. Preprint at https://arxiv.org/abs/2104.08042v1 (2021).

38 Gupta, R. et al. Microscopic evidence for anisotropic multigap superconductivity in the $\mathrm{CsV}_{3} \mathrm{Sb}_{5}$ 
kagome superconductor. Preprint at https://arxiv.org/abs/2108.01574 (2021).

39 Gallais, Y. \& Paul, I. Charge nematicity and electronic Raman scattering in iron-based superconductors. C. R. Physique 17, 113 (2016).

40 Luo, J. et al. Star-of-David pattern charge density wave with additional modulation in the kagome superconductor $\mathrm{CsV}_{3} \mathrm{Sb}_{5}$ revealed by ${ }^{51} \mathrm{~V}-\mathrm{NMR}$ and ${ }^{121 / 123} \mathrm{Sb}-\mathrm{NQR}$. Preprint at https://arxiv.org/abs/2108.10263 (2021).

41 Massat, P. et al. Charge-induced nematicity in FeSe. Proc. Natl. Acad. Sci. USA 113, 9177 (2016).

42 Lin, Y.-P. \& Nandkishore, R. M. Kagome superconductors from Pomeranchuk fluctuations in charge density wave metals. Preprint at https://arxiv.org/abs/2107.09050 (2021).

43 Tazai, R., Yamakawa, Y., Onari, S. \& Kontani, H. Mechanism of exotic density-wave and beyond-Migdal unconventional superconductivity in kagome metal $\mathrm{AV}_{3} \mathrm{Sb}_{5}(\mathrm{~A}=\mathrm{K}, \mathrm{Rb}, \mathrm{Cs})$. Preprint at https://arxiv.org/abs/2107.05372 (2021).

44 Balkanski, M., Wallis, R. F. \& Haro, E. Anharmonic effects in light scattering due to optical phonons in silicon. Phys. Rev. B 28, 1928 (1983).

\section{SUPPLEMENTARY}

In Supplementary Fig. S1 we present the raw, as-measured Raman scattering intensity for the in-plane scattering configuration $\left(\mathrm{A}_{1 g}+\mathrm{E}_{2 g}\right.$; panel a), as well as for the out-of-plane scattering configuration ( $\mathrm{E}_{1 g}$, panel b), covering the temperature range $5 \mathrm{~K}-300 \mathrm{~K}$.

The detailed inverse dynamic Raman susceptibility $1 / \chi^{\text {dyn }}$ is shown in Fig. S2 for both inplane (red squares) and out-of-plane (blue circles) configurations. The Curie-Weiss behavior is indicated by the dashed lines, yielding the Curie-Weiss temperatures of $T_{\mathrm{CW}}^{\mathrm{op}}=-96 \mathrm{~K}$ and $T_{\mathrm{CW}}^{\mathrm{ip}}=-22 \mathrm{~K}$ for the out-of-plane and in-plane scattering geometries, respectively.

The full set of collision-dominated fits to the Bose corrected Raman intensity $\chi^{\prime \prime}$ at all measured temperatures is plotted in Fig. S3 for the in-plane polarization, and in Fig. S4 for the out-of-plane polarization. For these fits, the data points in spectral ranges dominated by other excitiations (phonons, as well as the electronic continuum centered around $17 \mathrm{meV}$; indicated by the red-colored symbols) were neglected.

In Fig. S5 we plot the angular dependence of the as-measured Raman intensity recorded 

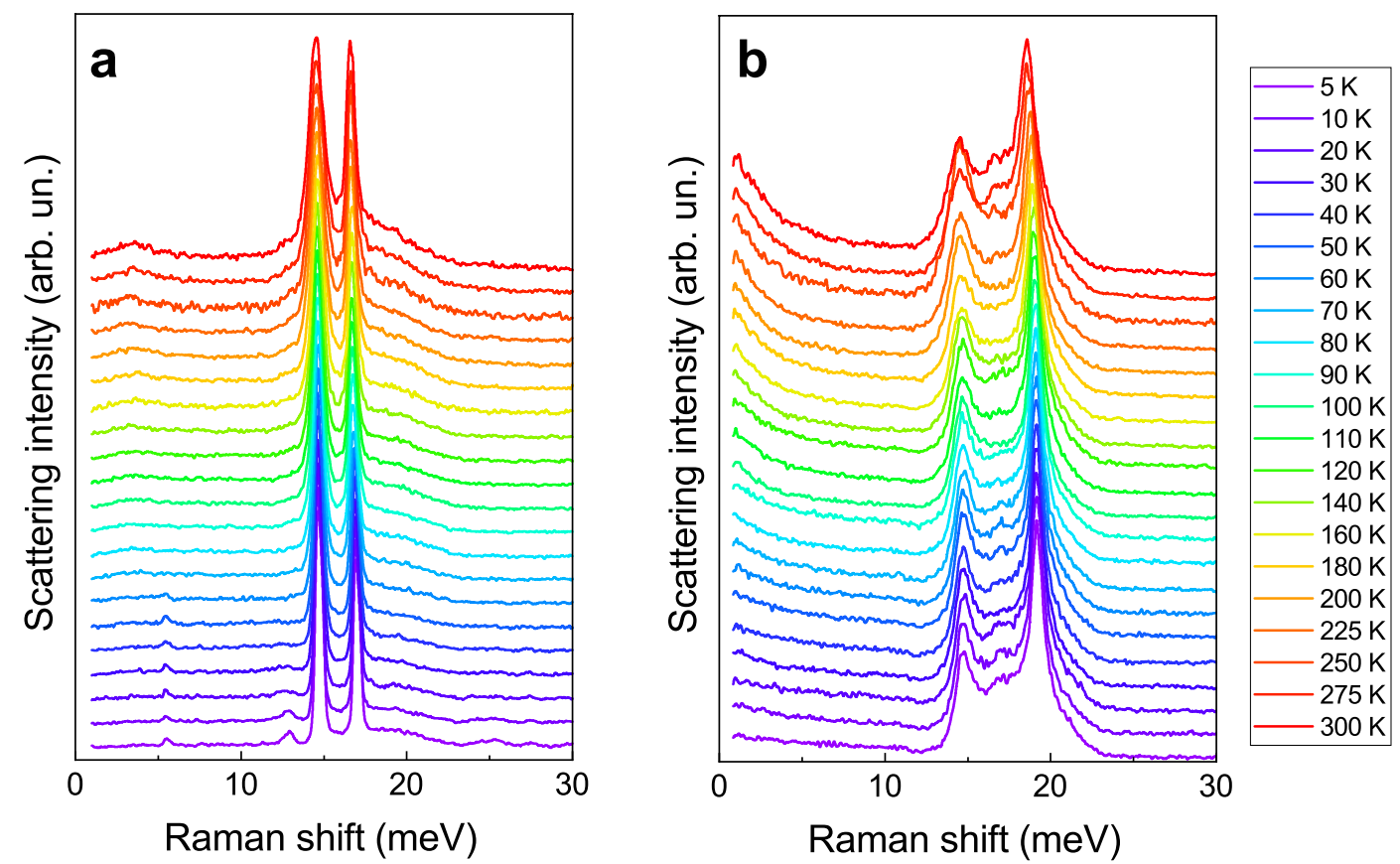

FIG. 5. S1: Temperature dependence of as-measured Raman intensity. a Raman scattering intensity measured in in-plane $\mathrm{A}_{1 g}+\mathrm{E}_{2 g}$ configuration. b Raman intensity measured in out-of-plane $\mathrm{E}_{1 g}$ configuration. Spectra are stacked in intensity for clarity.

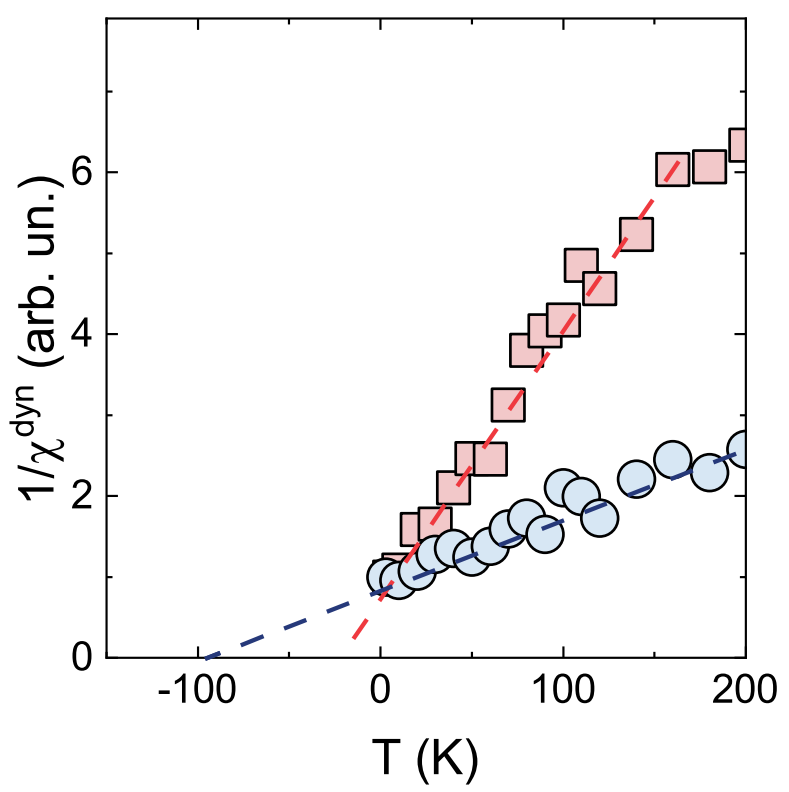

FIG. 6. S2: Curie-Weiss fits to the dynamic Raman susceptibility. The inverse dynamic Raman susceptibility $1 / \chi^{\text {dyn }}$ measured in-plane (squares) and out-of-plane (circles). Dashed lines indicate the linear Curie-Weiss behavior. 

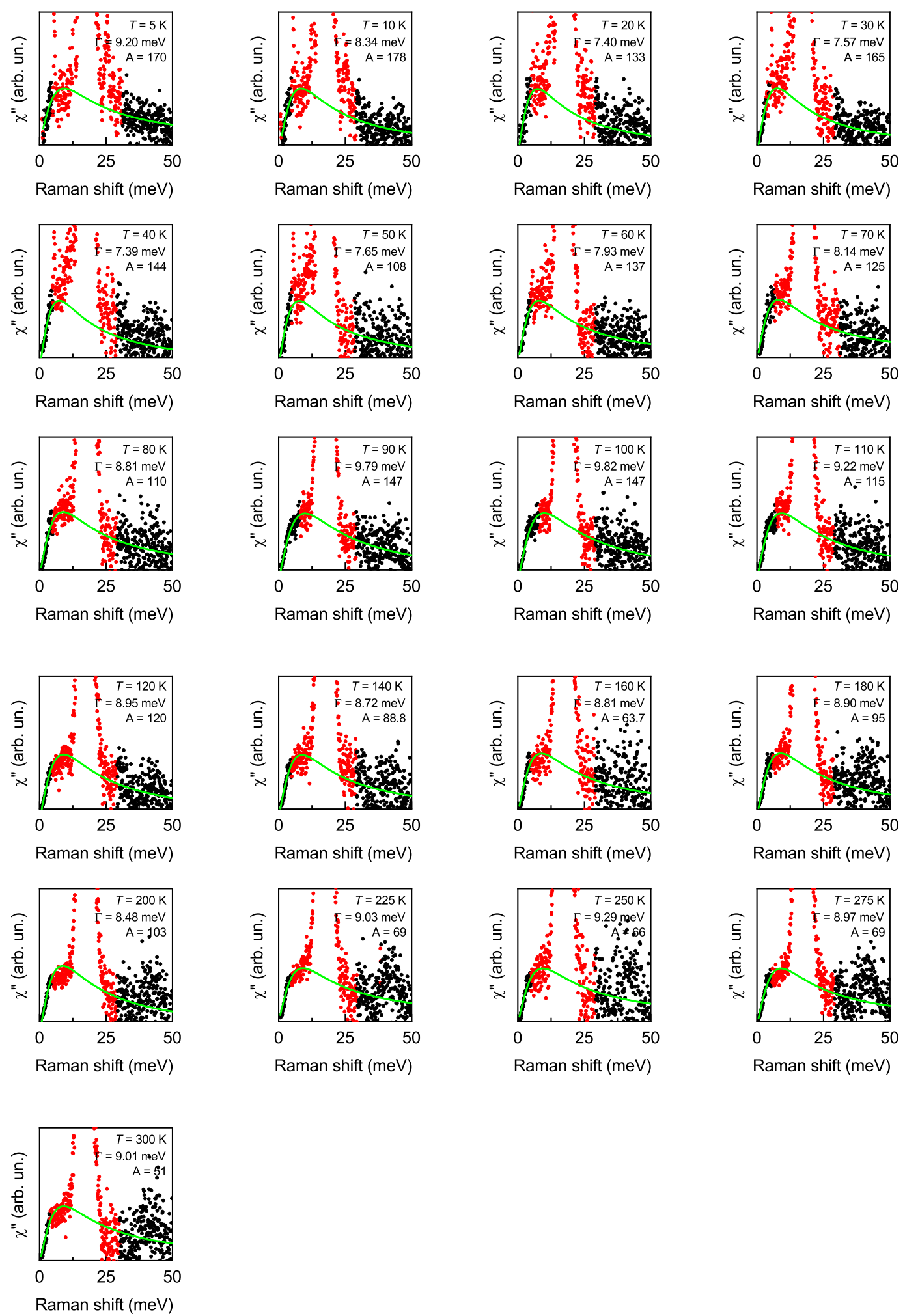

FIG. 7. S3: In-plane collision-dominated fits. Bose-corrected Raman response $\chi^{\prime \prime}$ (black and red symbols) measured in the in-plane $\mathrm{A}_{1 g}+\mathrm{E}_{2 g}$ channel over a temperature range from $5 \mathrm{~K}-300$ K. Green lines denote the collision-dominated fit, for which the black data symbols were considered. The red symbols denote the masked spectral range dominated by other excitations. The resulting fitting parameters $\Gamma$ and $A$ are indicated in each panel. 


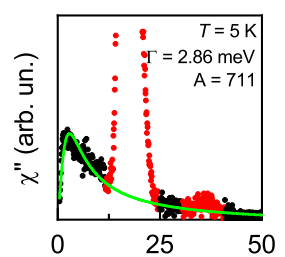

Raman shift (meV)

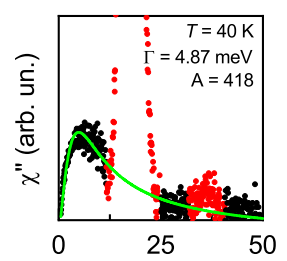

Raman shift (meV)

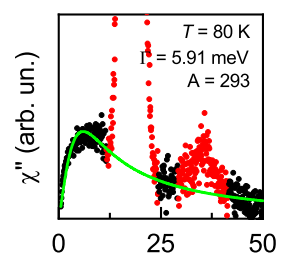

Raman shift (meV)
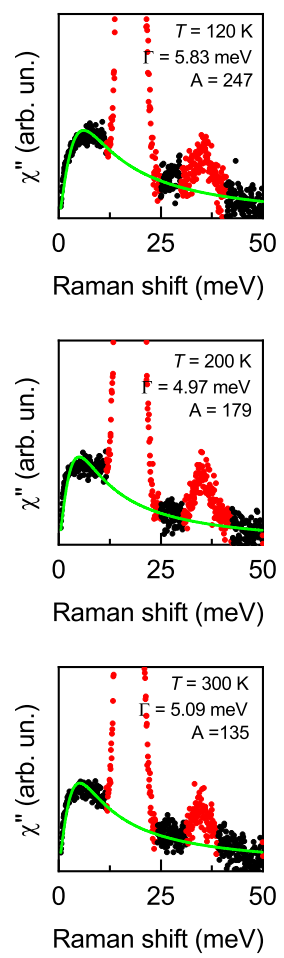
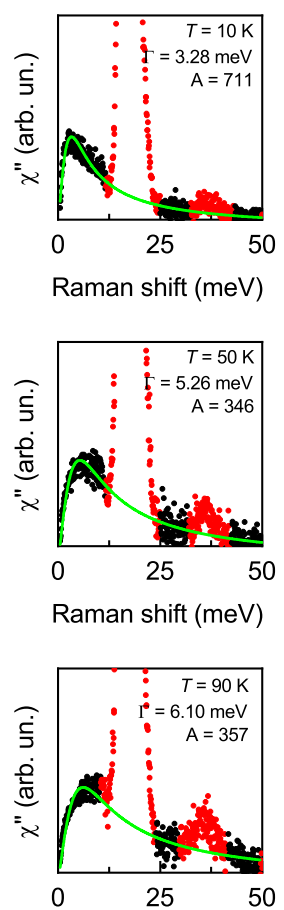

Raman shift (meV)

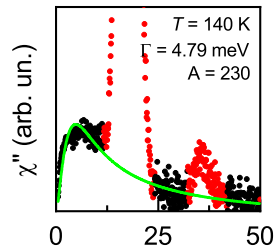

Raman shift (meV)

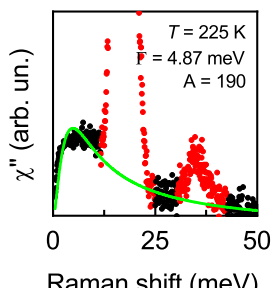

Raman shift (meV)

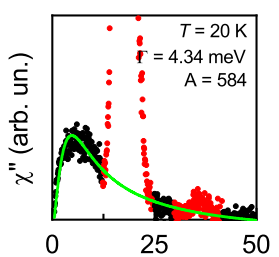

Raman shift (meV)

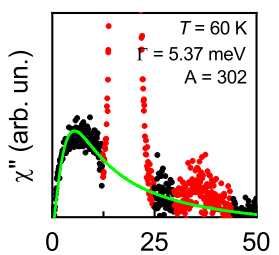

Raman shift (meV)

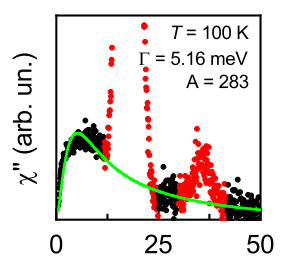

Raman shift (meV)
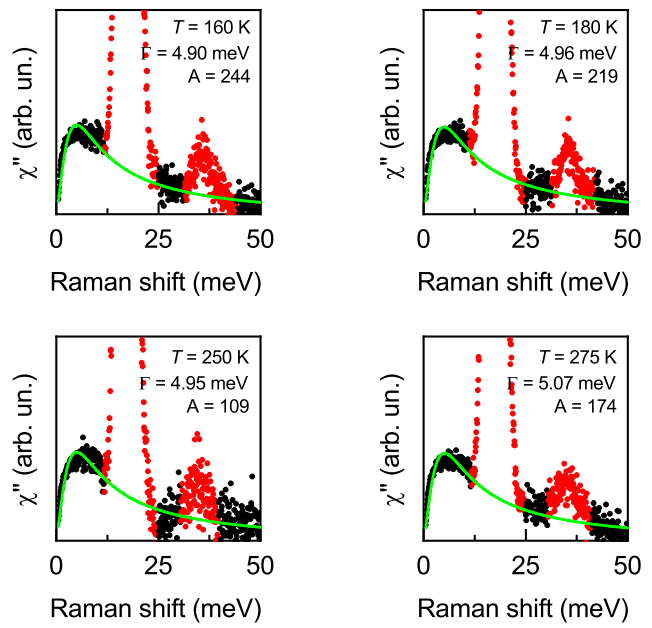

Raman shift (meV)

FIG. 8. S4: Out-of-plane collision-dominated fits. Bose-corrected Raman response $\chi^{\prime \prime}$ (black and red symbols) measured in the out-of-plane $\mathrm{E}_{1 g}$ channel over a temperature range from $5 \mathrm{~K}$ - 300 K. Green lines denote the collision-dominated fit, for which the black data symbols were considered. The red symbols denote the masked spectral range dominated by other excitations. The resulting fitting parameters $\Gamma$ and $A$ are indicated in each panel. 

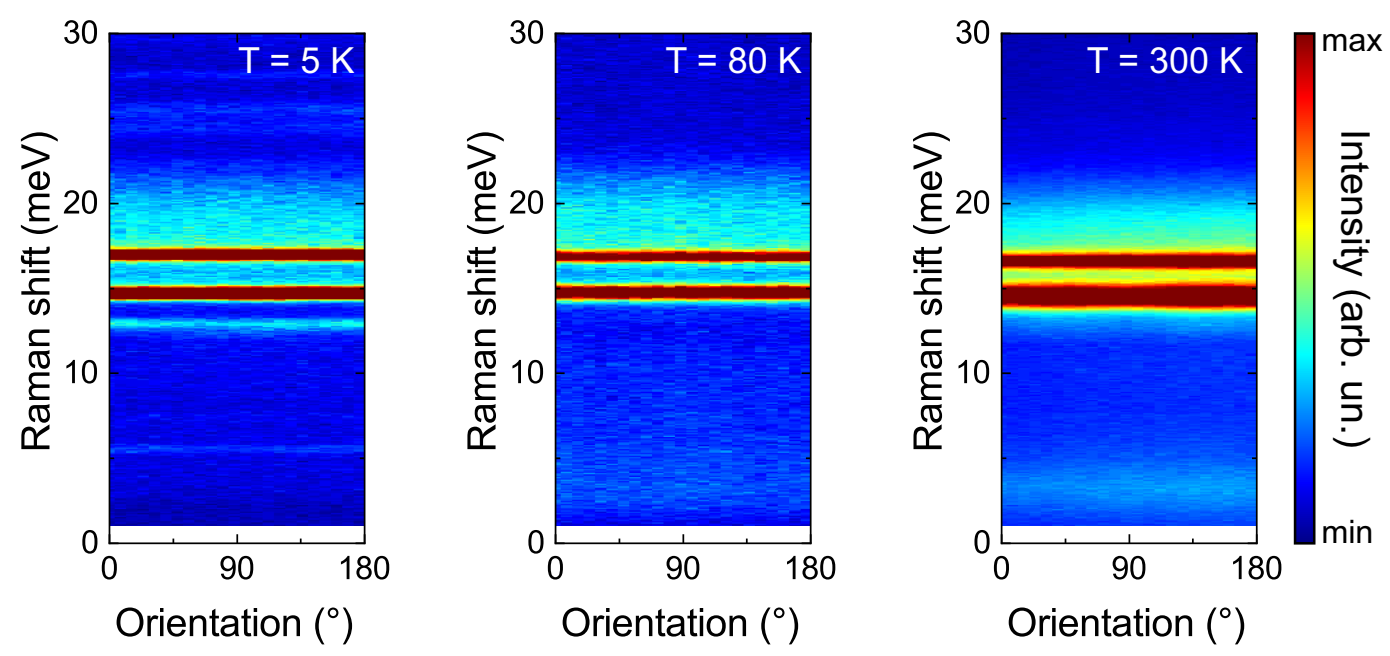

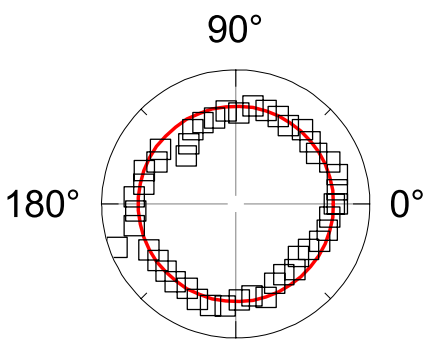

$270^{\circ}$

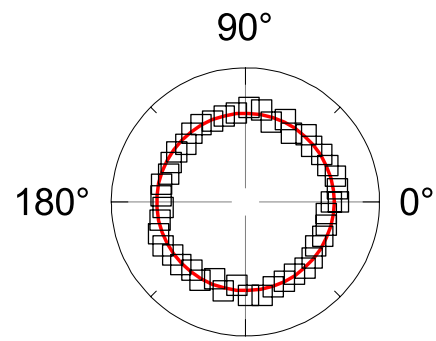

$270^{\circ}$

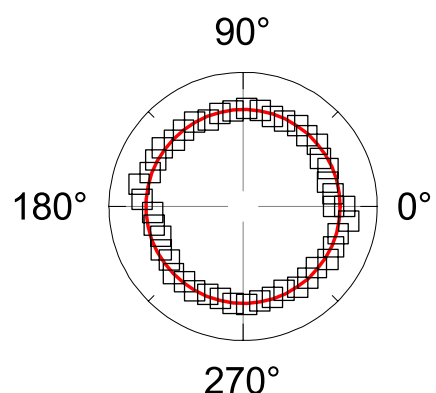

FIG. 9. S5: Angular dependence at various temperatures. Raman scattering intensity $I(\omega)$ obtained in in-plane configuration with the polarization of the light rotated in-plane over a range from $0^{\circ}$ to $180^{\circ}$ at fixed temperatures of $5 \mathrm{~K}, 80 \mathrm{~K}$, and $300 \mathrm{~K}$.

in the in-plane polarization over a range of $0^{\circ}-180^{\circ}$ at $T=5 \mathrm{~K}$ (i.e., close to $T_{c}$ ), at $T=80$ $\mathrm{K}$ (i.e., below $\left.T_{\mathrm{CDW}}\right)$, and at $T=300 \mathrm{~K}\left(T \gg T_{\mathrm{CDW}}\right)$. As seen from these three plots, we cannot resolve rotational symmetry breaking for phonons, charge-density-wave modes, as well as for electronic Raman scattering. Our angular variation data demonstrate that $s$-wave-like symmetry is largely retained for the electronic phases, while alluding to a subtle nature of a rotational symmetry broken state. 